\title{
Piezoelectricity in non-nitride III-V nanowires: Challenges and Opportunities
}

\author{
Yonatan Calahorra ${ }^{1}$, Sohini Kar-Narayan ${ }^{1 *}$ \\ ${ }^{1}$ Department of Materials Science and Metallurgy, University of Cambridge, 27 Charles Babbage \\ Cambridge CB3 OFS, UK
}

*Email:sk568@cam.ac.uk

\begin{abstract}
The increasing demand for portable and low-power electronics for applications in selfpowered devices and sensors has spurred interest in the development of efficient piezoelectric materials, by which mechanical energy from ambient vibrations can be transformed into electrical energy for autonomous devices and/or be used in strain-sensitive applications. Semiconducting piezoelectric materials are ideal candidates in the emerging field of piezotronics and piezo-phototronics, where the development of a piezo-potential in response to stress/strain can be used to tune the band structure of the semiconductor, and hence its electronic and/or optical properties. Furthermore, research into nanowires of these materials has intensified due to the enhancement of piezoelectric properties at the nanoscale. In this regard, nanowires of $\mathrm{ZnO}$ and the III-nitrides have been extensively studied, but the piezoelectric properties of non-nitride III-V semiconductor nanowires remain less-explored. Indeed, direct measurements of the piezoelectric properties of single III-V nanowires are tellingly rare due to the difficulties associated with measurements of piezoelectric properties of nanoscale objects using conventional scanning probe microscopy techniques. This review addresses the challenges related to the study of piezoelectricity in III-V nanowires and the opportunities that lie therein in terms of device applications.
\end{abstract}

\section{Introduction:}

\subsection{Background}

We begin by exploring the origin of piezoelectricity in non-nitride III-V nanowires (referred to simply as III-V nanowires from here onwards). The piezoelectric effect arises as a result of an applied stress causing a relative displacement of ions or polar components within a piezoelectric material, resulting in a change in net polarisation. Piezoelectricity thus refers to the linear interaction between the mechanical and the electrical states of certain materials where the stress and strain are interrelated with the resulting electric displacement induced in the material. Among the 32 crystal classes, 11 are centrosymmetric and non-polar such that an applied stress results in symmetrical ionic displacement and no net dipole moment. Of the 21 non-centrosymmetric classes, 20 are piezoelectric with the exception being the 432 cubic system. Piezoelectricity manifests as both the direct piezoelectric effect, where a polarisation is created in response to an applied stress, and the converse piezoelectric effect, where an applied electric field creates a strain within the material. The effect can be described mathematically with a set of complimentary equations given below: 


$$
\begin{aligned}
& D=\varepsilon_{T} E+d T \\
& S=d^{t} E+s_{E} T
\end{aligned}
$$

Here, $T$ and $S$ represent stress and strain respectively, which are second rank tensors related to an applied force and the normal to the area over which it acts. $D$ and $E$ represent the electric displacement and electric field which are vectors. $\varepsilon_{T}$ is the dielectric permittivity of the material at constant stress, $d$ is the piezoelectric tensor and $s_{E}$ is the elastic compliance at constant electric field. These three terms are represented by second, third and fourth rank tensors respectively, where $d^{t}$ is the transpose of $d$. Voigt notation is commonly used to simplify the tensors since static equilibrium requires $T_{\mathrm{ij}}=T_{\mathrm{ji}}, S_{\mathrm{ij}}=S_{\mathrm{ji}}$ and $s_{\mathrm{ijkl}}=s_{\mathrm{jjlk}}=s_{\mathrm{jikl}}=s_{\mathrm{jilk}}$, and further simplifications may arise when the $\varepsilon, d$ and $s$ tensors have symmetries for certain directions. Bulk III-V semiconductor materials with the non-centrosymmetric zinc blende (ZB) crystal structure possess off-diagonal "shear" piezoelectric components, while III-V nanowires of the same material, that more likely exhibit the wurtzite (WZ) crystal structure, exhibit distinct axial piezoelectric components (1). III-V semiconductors therefore present an interesting material system in which to investigate and possibly manipulate piezoelectricity, based on a combination of dimensionality, geometry, and crystal structure.

The past decade has seen a fast-growing scientific interest in piezoelectric applications based on nanomaterials, particularly following the pioneering work from Z.L Wang's group regarding ZnO nanowires (NWs) and nanoribbons (2-4), and their applications in mechanical energy harvesting. Piezoelectricity in II-VI materials such as $\mathrm{ZnO}$ and III-Ns is well documented $(5,6)$, and accordingly, significant research efforts have been directed towards understanding and utilizing piezoelectricity in NWs of these materials (2, 4, 7-10). GaN NWs (8) and $\mathrm{ZnO}$ nanobelts (2) have been examined via piezo-response force microscopy (PFM), and were found to have an increased piezoelectric response as compared to the bulk, possibly due to reduced mechanical constraints. Single NWs, as well as NW ensembles have been used to realize mechanical energy harvesting devices, as well as piezo-controlled diodes and transistors $(3,4,10-12)$. Notably, the term "piezotronic" was coined to describe the unique effects arising when semiconductor junctions (Schottky or p-n) are subject to strain (11-15).

Figure 1a shows the annual number of publications revolving around the topic of "Nanogenerator" and "Piezoelectric Nanogenerator" which concerns the ability to harvest ambient mechanical energy and transform it to useful electrical energy. Indeed, about $50 \%$ of publications revolving around this topic, deal with piezoelectric properties at the nanoscale. The interest in nanoscale materials is older than that, with NWs (having diameters of several nanometres to a few hundred nanometres) having been widely studied (16-18). III-V semiconductor nanowires are at the focus of 
(a)

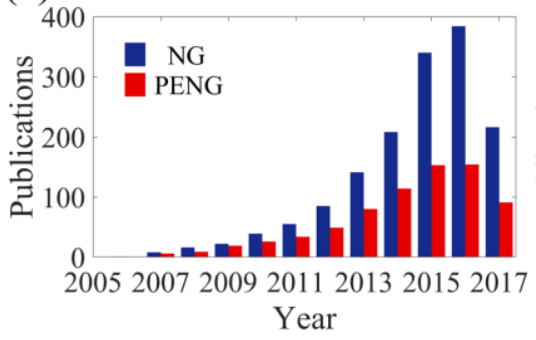

(b)

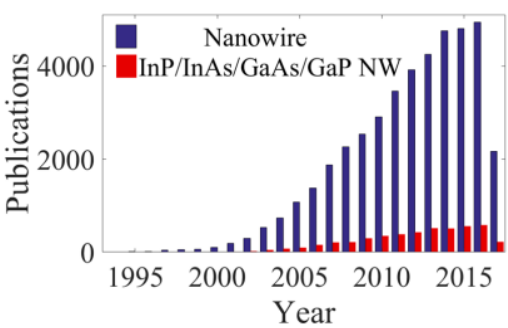

(c)

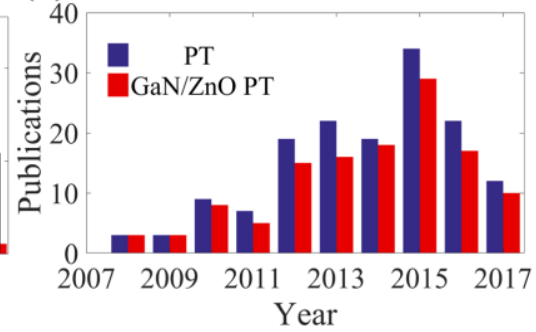

Figure 1b showing publications revolving around the topic of "Nanowires" and (a few non-nitride) "III-V Nanowires".

In addition to energy harvesting, traditional applications of piezoelectric materials, such as sensing applications, have been widely studied in $\mathrm{ZnO}$ and $\mathrm{GaN}$ based nanomaterials $(4,11,13)$. Considering the electrical and optical properties of semiconductors, the terms "piezotronics" and "piezophototronics" has been coined to respectively describe the electromechanical and optoelectromechanical interactions taking place in piezoelectric semiconductor junction based devices. This is an emerging research field with many potential sensing and energy-based applications, that is currently based on a handful of candidate materials systems. Further development of the field would require investigation and development of a wide range of piezoelectric semiconductors, including III-V materials which exhibit a wider range of bandgaps and mechanical properties that may be appropriate for specific applications. A schematic of the metal-piezoelectric semiconductor piezotronic effect is shown in Figure 2. Briefly, when a depleted piezoelectric semiconducting region experiences stress, a negative or positive piezo-potential (dependent upon the crystal orientation and the sign of the resulting strain) will result in additional interface charge. This charge is not neutralized, considering that the material is depleted of charge carriers, and therefore serves to modify the Schottky barrier height. Taking into account the optoelectronic properties of Schottky junctions, the piezophototronic effect relates to such junctions when light is shone upon them. To elucidate piezoelectric semiconductor related research,

(a)

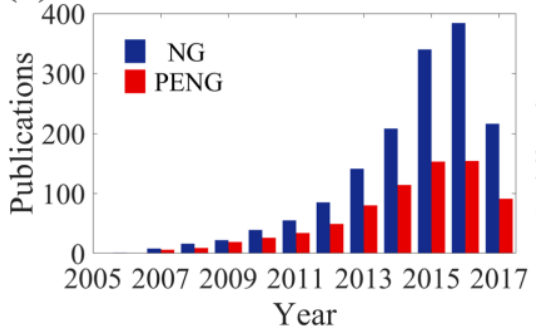

(b)

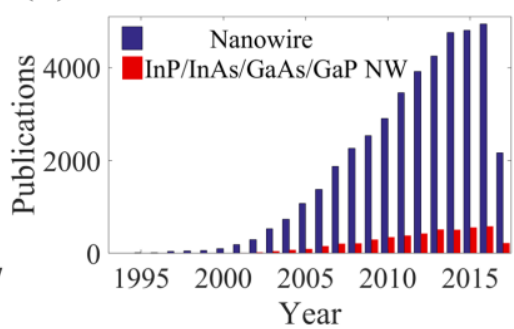

(c)

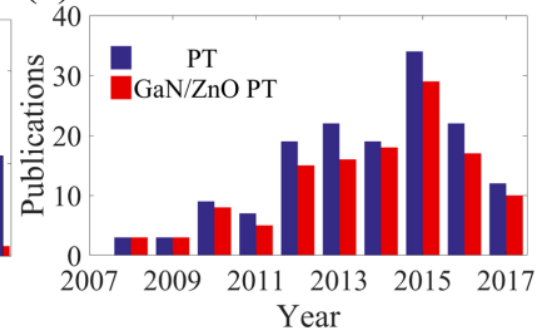


Figure 1c shows the annual number of publications revolving around "piezotronic" and "GaN/ZnO piezotronic". Indeed, GaN and $\mathrm{ZnO}$ related publications comprise the majority of papers, while nonnitride III-V nanomaterials have hardly been studied in the context of piezotronics. In fact, a Web of Science topical search of "InP/GaAs/InAs/GaP piezotronic" yields only 5 results - none of which actually deal with III-V materials.

The relative lack of interest in III-V NW piezoelectricity is intriguing considering the closeness in applications as well as in underlying physics of III-Vs and III-Ns $(19,20)$. One possible reason for this might be the smaller piezoelectric coefficients associated with non-nitride III-V materials $(19,20)$. However, these are not orders of magnitude apart ( $5 \mathrm{pm} / \mathrm{V}$ for III-Ns, $\sim 1 \mathrm{pm} / \mathrm{V}$ for III-Vs), and moreover, with the reported increase in effective piezoelectric coefficients in NWs as compared to bulk (8), research into III-V NW piezoelectric properties and applications may be of considerable interest. Another reason why piezoelectric properties of III-V NWs have been rarely studied may be due to the predisposition of the III-V community towards pure electronic and optical applications, as well as the inherited knowledge from the vast world of III-V bulk and epitaxial thin films. Here, the dominant crystal growth orientation is $\{100\}$ (e.g., see Ref. (21)), and the dominant crystal structure for III-V materials is zinc-blende ( $\mathrm{ZB})$, where spontaneous electrical polarization is negligible, and piezoelectricity is limited to three shear coefficients (19). Therefore, although known, recognized, and used to some extent for micro-electromechanical systems (MEMS) (22, 23), polarization and piezoelectricity do not play a significant role in traditional III-V technology. This stands in striking contrast to GaN technology where the dominant crystal structure is wurtzite, a considerable degree of spontaneous electrical polarization exists, and the dominant growth orientation is actually the polar $\{0001\}$ (equivalent to $\{111\}$ in ZB structure). For example, GaN high-electron mobility transistor (HEMT) technology is physically based on the spontaneous and piezoelectric polarization mismatch related to III-N heterostructures (20). Hence, there has been understandably more awareness and interest in nanoscale semiconductor piezoelectricity from the III-N scientific community, than from the III-V community. However, as we will explain in the first few sections of this review, III-V NWs have a lot in common with the III-N point-of-view when it comes to piezoelectricity, and in light of the growing interest in semiconductor piezo-electronics and the vast interest in III-V NWs, there exists tremendous scope to explore and exploit the piezoelectric properties of III-V NWs.

This review is structured as follows: in the remainder of Section 1 we briefly review piezoelectricity and related applications in bulk III-V materials, as well as the key distinctions between III-V bulk and NWs in terms of crystalline structure. In Section 2 we review the current status of NW mechanical properties, with emphasis on studies dedicated to WZ III-V NWs. In Section 3 we will review the body of work related to piezoelectric and electromechanical effects in III-V NWs, considering three aspects: piezoelectricity related to internal stresses, piezoelectricity related to external mechanical forces, and finally direct observations of fundamental piezoelectricity in III-V NWs. In 
Section 4 we conclude and point out the challenges lying ahead in this research direction. The review considers both theoretical and experimental results, and therefore when relevant, comparisons made between experiments and theory will be discussed. However, while some of the experimental set-ups and results will be examined and compared, a thorough discussion of theoretical methods for calculating mechanical/piezoelectric properties and related intricacies are outside the scope of this review.

\subsection{Piezoelectricity in bulk III-V materials}

Although not playing a significant role in the design and analysis of electronic devices, piezoelectricity arising from the non-centrosymmetric ZB structure in III-V semiconductors has been long known $(24,25)$. III-V materials exhibit shear piezoelectricity (relative to the basic cubic cell vectors), with an identical value for all three orientations (see Eq. 1). This property has been utilized to demonstrate electromechanical applications $(22,23,26,27)$. Furthermore, the effect of internal piezoelectric fields on the performance of $\{111\}$ oriented electronic devices has been considered (28, 29).

Notably, some early studies which are closely related to current piezoelectric-NW studies have gone relatively unnoticed. Two interesting studies are those by Kasuka et al. which represent an early demonstration of the piezotronic effect (albeit, prior to coining the term) on both p-type and n-type $\{111\}$ oriented GaP Schottky diodes $(30,31)$. In these two papers, Kasuka and co-workers have shown that the effect $\{111\}$-oriented strain has on the Schottky barrier height is sensitive both to carrier polarity (electrons/holes), as well as crystal face $(\mathrm{Ga} / \mathrm{P})$ contacting the metal. Furthermore, as would be expected, in case of compression the trends were mirrored. An electro-mechanical analytical analysis (considering stress, electrical displacement, and Poisson's equation) provided an inversely linear relation between the barrier height and the strain. Bearing in mind that for diodes the current is exponentially dependent on barrier height, this has implications for the strain-sensitivity of piezotronic effect. It is worth revisiting Figure 2 to qualitatively understand these effects. Figure $2 \mathrm{a}$ and $2 \mathrm{~b}$ show an n-type Schottky contact subjected to compressive and tensile stresses, correspondingly. These stresses result in opposite polarization charges at the interface, hence the opposite effect on the barrier and current. The semiconductor polarity will also have a similar effect: while for $\mathrm{n}$-type band bending upwards (compression in this example) results in increase of the barrier, for p-type (of the same polarity) the same mechanical input will decreases the barrier.

In a recent work, seeking to develop magnetoelectric composites, Gerngross et al. have examined the piezoelectric properties of porous InP substrates (ZB), obtained by chemical etching (32). Interestingly, they found that the piezoelectric strain coefficient (measured optically) was up to 30 times larger than the literature value, depending on the details of the etching process. They attributed the effect to reduced free-carrier screening of the piezo-generated-voltage, arising from increased surfacerelated depletion of the porous structure. We further suggest that in addition to depletion, the porous 
material is softer, giving rise to further enhancement to the measured coefficient, similar to reports for enhanced mechano-electric coupling in porous PVDF (polyvinylidene fluoride) (33).

If so, we have seen in the above that piezoelectricity in III-Vs has in fact been considered in the past, and examined both experimentally and theoretically in different ways for various applications. Nevertheless, this trend has not been widely followed; the main reason probably being the tendency to prefer the $\{110\}$ and $\{100\}$ planar growth directions over the polar $\{111\}$ direction, due to practical difficulties associated with the growth of thin films. In sharp contrast, $\{111\}$ planes and orientations are the default working environment for III-V NWs, as discussed below, and this should motivate revisiting this aspect of III-Vs and its relevance to piezoelectricity when considering NWs.

\subsection{The effect of growth orientation and crystal structure on III-V NW Piezoelectricity}

III-V NWs may by realized by either bottom-up or top-down approaches. Although top-down NWs may represent the next generation of electronic devices $(34,35)$, bottom-up NWs introduce interesting physical phenomena due to the realization using crystal growth methods $(16,36,37)$. Bottom-up III-V NWs are usually grown using a nano-sized metal catalyst lying on the growth substrate, which can be either silicon, or a bulk III-V substrate $(36,38)$. Nanowires can also be grown using catalyst-free methods, where a nano-sized pore within a growth-selective mask facilitates the growth of a NW (39). A few intermediate methods exist, combining characteristics of both; these include self-catalysed grown NWs, where inside the pore a non-foreign mantellic particle catalyses the growth (usually group-III metal) (40), and catalyst assisted selective-area NW growth, where the metal catalyst is deposited within a pore in a selective area mask $(41,42)$.

Although many unique properties arise from the various growth regimes (43-46), there are common traits to all: due to energy considerations, cubic III-V NWs are most likely grown axially in the $\{111\}$ (ZB) orientation (36). Consequently, the majority of III-V NW related work is based on $\{111\}$ substrates (either III-V or silicon), whereby NWs are vertically grown on these surfaces. Furthermore, there is an increased probability for NW growth in the WZ crystalline structure (36, 44, 47-49), in the $\{0001\}$ orientation, also vertically aligned on $\{111\}$ growth substrates. As will be seen below, both these properties indicate that studying piezoelectric properties of III-V NWs might be beneficial. Figure 3a shows an example of sharp crystal phase heterostructures in various III-V NWs (44). Next, an abrupt heterostructure is shown both experimentally (Figure 3b, single GaAs NW TEM), and schematically (Figure 3c).

As mentioned earlier, ZB III-V materials exhibit only shear piezoelectricity, rendering the piezoelectric strain tensor (using Voigt notation ) (19) 


$$
d_{Z B,<001>}=\left(\begin{array}{cccccc}
0 & 0 & 0 & d_{14} & 0 & 0 \\
0 & 0 & 0 & 0 & d_{14} & 0 \\
0 & 0 & 0 & 0 & 0 & d_{14}
\end{array}\right)
$$

where $d_{14}$ is a shear piezoelectric coefficient. The piezoelectric effect and tensor notations have been widely discussed in the literature, for example see Ref. (50).

In NWs, where the dominant axial orientation is $\{111\}$, it is beneficial to rotate the tensor such that the piezoelectric axes correspond with sample geometry $(1,51,52)$. Additionally, to chemically conform with WZ (53), we choose the 3-axis to be $\langle 111\rangle$, corresponding to $\langle 0001\rangle$ in WZ, the 1-axis to be $\langle 11-2\rangle$ and 2-axis to be $\langle-110\rangle$ (corresponding to $\langle 10-10\rangle$ and $\langle-12-10\rangle$ in WZ, respectively). The resulting matrix is (1)

$$
d_{Z B,<111>}=\left(\begin{array}{cccccc}
d_{11} & -d_{11} & 0 & 0 & d_{15} & 0 \\
0 & 0 & 0 & d_{15} & 0 & d_{26} \\
d_{31} & d_{31} & d_{33} & 0 & 0 & 0
\end{array}\right)
$$

The values of the coefficients are then $-d_{15}=d_{33}=\sqrt{\frac{1}{3}} \widetilde{d_{14}}, d_{31}=\frac{1}{2} d_{15}, d_{11}=-\sqrt{\frac{1}{6}} \widetilde{d_{14}}$ and $d_{26}=$ $\sqrt{\frac{2}{3}} \widetilde{\mathrm{d}_{14}}$, where $\widetilde{\mathrm{d}_{14}}$ is the shear value from the original tensor (Eq. 3).

In other cases, NWs crystallize in WZ structure with $\{0001\}$ axial orientation, and the piezoelectric matrix is

$$
d_{W Z,<0001>}=\left(\begin{array}{cccccc}
0 & 0 & 0 & 0 & d_{15} & 0 \\
0 & 0 & 0 & d_{15} & 0 & 0 \\
d_{31} & d_{31} & d_{33} & 0 & 0 & 0
\end{array}\right)
$$

As evidenced above, in their most common forms, III-V NWs are expected to exhibit distinct piezoelectricity compared to bulk, due to geometry, crystal orientation and crystal structure.

We list in Table I characteristic piezoelectric strain coefficients for a few III-V materials, GaN and $\mathrm{ZnO}$. Note that while the $\mathrm{ZB}$ values are experimentally verified, the WZ values for III-Vs are theoretical, obtained using ab-initio calculations (AI) (54), or by applying a quasi-cubic (QC) model, yielding $\mathrm{e}_{14, \mathrm{ZB}}=\sqrt{\frac{1}{3}} \mathrm{e}_{33, \mathrm{WZ}}(25)$, and $\mathrm{e}_{31}=-\frac{1}{2} \mathrm{e}_{33}$ (55). The relations between piezoelectric charge coefficients $\left(e_{i j}\right)$ and piezoelectric strain coefficients $\left(d_{i j}\right)$ follow (24) $e_{14}=d_{14} c_{44}$ for $Z B$, and

$$
\begin{gathered}
e_{31}=d_{31}\left(c_{11}+c_{12}\right)+d_{33} c_{13} \\
e_{33}=2 d_{31} c_{13}+d_{33} c_{33}
\end{gathered}
$$

where $c_{i j}$ are stiffness coefficients (56). $e_{i j}$ coefficients are in $\left(C / \mathrm{m}^{2}\right) ; d_{i j}$ coefficients are in $(\mathrm{pm} / \mathrm{V})$.

If so, we can see that the theoretical predictions for the piezoelectric coefficients in WZ structure vary greatly. Since all of these NWs are routinely grown, it should be possible to experimentally measure these quantities, validating existing theories and shedding light on possible applications. Notice that $\mathrm{e}_{\mathrm{ij}}$ and $\mathrm{d}_{\mathrm{ij}}$ coefficients do not necessarily follow similar trends, this is due to their co-relation incorporating the stiffness of the material. 


\section{Mechanical properties of WZ III-Vs}

The interest in III-V NWs ushered the emergence of WZ III-V materials. The immediate implication was that the most basic physical properties became an interesting research topic both theoretically and experimentally. Surprisingly, the mechanical properties of WZ III-V NWs received little attention, compared to the vast interest in their optical properties $(17,18,57)$, or the general interest in NW mechanical properties $(58,59)$. Nevertheless, in recent years, researchers have begun directly looking into this topic, either by applying nanomechanical methods using atomic force microscopy (AFM) $(60,61)$, in-situ transmission electron microscopy (TEM) setups (62-64), or optical methods (65).

Figure 4 shows the results of mechanical characterization of WZ GaAs and InP NWs, obtained by the various methods mentioned above. Figure 4a shows the Young's modulus obtained by Chen $e t$ $a l$. by buckling tests (see inset) conducted on pure WZ and mixed phase GaAs NWs as reported in Ref. (64); similar measurements have been conducted for ZB NWs as well (62). In both cases, a significant increase in the elastic modulus with decreasing diameter is found, and these findings were explained by the elastic core-shell model, where the GaAs native oxide exhibits a high elastic modulus, shifting the composite effective value upwards with size reduction. An additional result of this study was that the presence of stacking faults was associated with an additional increase in Young's modulus (red and black curves in Figure 4a). In other studies, using optical measurements of WZ (and ZB) GaAs (Figure 4c) (65), as well is in AFM-based nanoindentation measurements of WZ InP NWs (Figure 4b) (60), the values obtained for WZ NWs were very close to theoretical predictions $(56,66)$; this was the case even for NWs thinner than those shown in the TEM-based studies. By further contrast, in the AFMbased study, stacking faults were associated with a considerable reduction of extracted modulus value, and not an increase.

Note that while both the AFM-based and optical studies took into account the inherent anisotropy of the elastic properties of III-V materials, the TEM-based studies did not, and thus have possibly induced misinterpretation of the results during modelling. Furthermore, the role of the native oxide in increasing the effective modulus with diameter reduction, requires further validation, e.g., by studies of NW with an enhanced oxide layer, or with no oxide layer (58). Indeed, in different studies, researchers have correlated stacking faults and surface oxide with a reduced Young's modulus (as compared to theoretical values) of WZ and mixed phase InAs NWs $(67,68)$.

While there is a definite need for further study of the basic mechanical properties of III-V NWs, and in particular the role of stacking faults, it seems that for NWs thicker than a few nanometres, the current theories provide an adequate basis for the assessment of the compliance/stiffness tensor and the subsequent elastic modulus, which represents a fundamental requirement for the analysis of piezoelectricity (56). 


\section{Electromechanical effects in III-V NWs}

Various researchers have considered the piezoelectric properties of III-V materials in forming NWs and NW heterostructures. The chronological advance of these studies shows earlier focus on effects resulting from internal strains in nano-heterostructures, moving on to examination of the effect of external mechanical manipulation on optical or electrical properties, and finally studies focusing on the core piezoelectric effect in III-V NWs.

\subsection{Electromechanical effects related to internal strain}

Several studies have calculated the effects of strain resulting from III-V NW heterostructures on its properties, with consideration of the piezoelectric effect in particular $(51,52,54,69,70)$, with earlier studies having considered similar effects in planar quantum dots $(71,72)$. Niquet and Mojica have performed band diagram calculations for InAs segments in InP NWs (and vice versa) (69). Interestingly, such earlier works considered ZB NWs, while more recent studies have started considering WZ as well. Faria Junior and Sipahi have calculated the band structures of WZ/ZB InP NW heterostructure, with consideration of spontaneous and piezoelectric polarization (70). Additional studies considering core-shell, embedded, or periodic heterostructures where crystal orientation and structure affect piezoelectric potential have been published as well $(51,52,54)$.

In addition to theoretical studies, several authors have used these strain-induced effects in order to explain experimental results (73-77). Zervos and Feiner have used the lattice-mismatch-induced strain in an axial InAs/InP NW-based resonant tunnelling diode, to model experimentally observed shifts in the tunnelling resonance in the two opposite current orientations. They have modelled the electric field resulting in the strained regions, giving rise to directionality in the structure (74) (see Figure 5a). Anufriev et al. have examined the photoluminescence of a WZ InAs quantum rod embedded within a WZ InP NW, and found results coinciding with the quantum confined Stark effect. Although neglecting spontaneous polarization in the materials, they have considered internal-strain related piezoelectric fields to model the results (75) (see Error! Reference source not found.b).

\subsection{Electromechanical effects related to external strain}

Next, we turn to examine the influence of externally applied mechanical stress/strain on the electronic/optoelectronic properties of III-V NWs (78-84). Existing studies have been concerned with the application of axial pressure on the optical properties of WZ (83) and ZB (84) GaAs NWs, and of hydrostatic pressure on the optical properties of WZ InP NWs (78). In general, these studies were focused on the effect of pressure on the NW band-gap, i.e. piezoresistivity. They all report considerable changes in the nature of light-matter interactions with applied pressure, brought about by different evolution of energy sub-bands with pressure. However, direct consideration of piezoelectric effects, in a similar manner done for heterostructure-related internal stresses, has not been addressed. 
Other studies have dealt with pressure dependent electronic transport. Li et al. have studied the effect of tensile and compressive axial strain (0-3\%) on the conduction of two terminal InAs NW-based devices, with $\mathrm{ZB} / \mathrm{WZ} /$ mixed phase structures as well as with various axial growth orientation (79). They have calculated the electromechanical gauge factor (relative change in conductance per unit strain) for each NW examined. Interestingly, the only non-negligible result arises when $<0001>$ WZ NWs are measured, while ZB NWs in $\langle 011\rangle,\langle 103\rangle$ and $<-2-11>$ orientations and non-polar $<11-20\rangle$ WZ NWs result in no observable electromechanical response. The highest electromechanical gauge factor was found for the pure <0001> WZ NWs, while it was found that stacking faults reduce the electromechanical responsivity. The gauge factor was found to have contributions from piezoresistivity (symmetrical with respect to applied voltage) and the piezotronic effect (asymmetrical even when identical electrodes were used - Figure 6a). We note that the negligible electromechanical response in non-polar WZ NWs and ZB NWs remains intriguing considering piezoresistivity is still expected.

Indeed, in a further study by Zheng et al., pure phase NWs ( $<0001>$ WZ and $\langle 110\rangle$ ZB) were subjected to compressive strains, and the electromechanical transport effects were examined (80). In their experiments the NWs exhibited ohmic behaviour, and so the piezotronic effect was excluded from the explanation, leaving behind piezoresistivity. In a complementary fashion to the previous study, it was found that compressive strain reduced the current in the WZ NW. Interestingly, for the ZB NWs the current was found to have increased, shedding light on the previously absent piezoresistivity in ZB NWs. Let us consider the differences between this study and the previous one, and between the ZB and WZ NW shown (Figure 6b). Firstly, the gauge factors considered by Li et al. are significantly larger, and therefore the smaller differences considered by Zheng et al. allow better resolution, indicating that indeed piezoresistivity exists for ZB as well as WZ NWs. Secondly, taking a closer look at the deformations of the WZ and ZB NWs, it seems that the WZ NW undergoes buckling, while the ZB NW undergoes bending. If that is the case, the stress release associated with the buckling may result in the return of the current to its original level. For the ZB NW, the bending may induce compression and tension on different parts of the NW, resulting in current enhancement (on one of the sides). Zheng $e t$ al. include a detailed crystal orientation related examination of the piezoresistivity in the NWs. Additional studies were performed on InAs (82) and InAsP (81) NWs. In both cases the piezoresistivity was examined and used to explain the electromechanical gauge factor. Specifically, for InAs NWs the pressure dependence of surface state energy was considered (82). An interesting pattern emerges when considering the previous two sections: when external mechanical stresses are applied, the majority of studies focus on piezoresistivity. This might be due to the fact that most transport studies focus on InAs, usually yielding ohmic contacts thereby rendering the piezotronic effect negligible. Furthermore, most of these studies focused on "simple" (junction-less, non-heterostructure) NWs, where polarization effects are again negligible. This is unlike the studies which considered junctions and explored internal strain-related piezoelectric effects. However, considering piezotronics using III-V NWs for future applications might still be beneficial. In re-examining the results by Li et al., which remain the single 
study to date dealing with III-V NW piezotronics, the largest gauge factor was associated with the piezotronic effect, while the piezoresistivity-related contribution was smaller (as seen in other studies). We offer an intuitive explanation for this: while fundamentally piezoelectricity and piezoresistivity are linear (disregarding higher order phenomena for now), the current across a semiconductor barrier is exponentially dependent upon its height. Therefore, it might be that piezotronic devices are inherently more sensitive compared to piezoresistive devices, indicating that further research in this direction is worthwhile.

\subsection{Direct examination of piezoelectricity in III-V NWs}

In the previous sections we have examined the application of internal piezoelectric field effects to explain asymmetries in the electrical conductance and photoluminescence of III-V NW heterostructures, and we have surveyed the current literature on external electromechanical effects in III-V NWs, mostly dedicated to piezoresistivity. In this section we focus on an aspect which is as fundamental as much as it is unavoidable for future consideration of piezoelectric-based III-V applications: the basic piezoelectric properties of III-V NWs. In this case, the body of work is even smaller than for the previous topics, consisting of four papers that we are aware of. Three of these deal with the piezoelectric properties of GaAs NW ensembles (85-87), and the other one, and the first study to-date to directly examine the piezoelectricity in single III-V (InP and GaAs) NWs, is our own recent publication (1).

In the first study by Soshnikov et al., a mixed phase (mostly WZ) GaAs NW ensemble was embedded in poly(methyl methacrylate) (PMMA) and contacted from top and bottom to fabricate a vertically aligned NW based capacitor structure (85), similar to typical piezoelectric nanogenerator (PENG) structures. The sample was subjected to acoustic excitation, and the generated voltage recorded. An effective $d_{33}$ value of about $22 \mathrm{pmV}^{-1}$ was extracted following setup calibration. These experimental results were further analysed, drawing attention to the order of magnitude increase in the extracted coefficient compared to the theoretically expected value for WZ GaAs $d_{33}$ of about $2.3 \mathrm{pmV}^{-1}$ $(86,87)$. Following finite element simulations, the dominant effect in the observed increase of the effective piezoelectric coefficient was attributed to "pressure force enhancement", a geometrical factor brought by the ratio between the top electrode surface area and the NW diameter. A similar factor related to the "fill-factor" of the NWs on the substrate is examined, where the effect of the PMMA material in between the NWs is demonstrated. The simulations successfully recreate the experimental trend of an optimum NW density for increasing the electromechanical coupling (Figure 7), and in manifesting the importance of geometry in determining the effective piezoelectric behaviour of a given configuration.

Recently we have examined single III-V NWs using a non-destructive piezoresponse force microscopy (PFM) apparatus (1) developed in our group (88). Briefly, PFM is a scanning probe technique, where an atomic force microscopy tip in contact mode applies an electrical field to a 
piezoelectric samples, thus inducing convers-piezoelectric deformations, which are picked up by the same tip (89). By superimposing an electrical signal on a mechanically oscillated tip probing the sample, we have realized a non-destructive PFM method (1). Specifically, mixed phase (predominantly ZB) GaAs NWs were measured in a horizontal configuration, while WZ InP NW stems were examined in a vertical configuration. Figure 8a shows a GaAs NW scanned across its length, and the related COMSOL computed deflection levels in three directions (out-of-plane, axial in-plane, transverse in-plane). It was found that the experimental vertical PFM signal obtained from the NW could be explained as a superposition of the simulated vertical deflection and the transverse in-plane deflection, coupled to the vertical sensor through cantilever buckling. Furthermore, the simulated axial in-plane deflection bore a resemblance to the measured lateral signal (in the axial direction). Figure 8b shows the results obtained for vertically oriented InP NWs, showing a clear distinction from the surroundings ( $\mathrm{SiN}_{\mathrm{x}}$ dielectric). Following calibration, a value of $1 \mathrm{pmV}^{-1}$ was extracted from this measurement, which is reasonable considering the known properties of InP. Interestingly, this value is 5 times larger than the minimal theoretical values obtained for InP, and 5 times smaller than the maximal value (see Table I). Nonetheless, further characterization is required to validate this result, and to decouple material intrinsic effect from geometrical effects in the NWs.

Both studies demonstrate how the system geometry has a significant influence on piezoelectric performance, either by device characteristics or measurement apparatus specifics (e.g., non-uniform field in a tip/NW/substrate configuration). These effects render the interpretation of experimental results challenging, conveying the importance of simulations as well as highlighting the necessity of further study in this topic for better understanding of fundamental and device-related piezoelectric performance.

Furthermore, both these studies indicate the piezoelectric coefficients at the 10-100 nm scale are similar to those theoretically predicted. This stands in some contrast to the results obtained for GaN NWs, where the axial piezoelectric strain coefficient $\left(d_{33}\right)$ was found to be 3-6 times higher than reported for bulk $(8,90)$. Minary-Jolandan et al. further reported a similar increase in the two other piezoelectric coefficients (8). Although we are unaware of a study directly comparing NWs and bulk material, Tiwary et al. went on to perform such finite element simulations (90). Their results indicate that the inherent softening of the NW compared to thin films (reduced mechanical constraints) might be behind the increased electromechanical response. In addition, first-principle studies predict the increase of effective piezoelectric coefficients at the extreme (1-10 nm) nanoscale $(91,92)$, however the application of these results to NWs an order of magnitude thicker would not be easily justified. In any case, the current picture arising form available data is that although further study of this topic is required, NW-based geometry might promote electromechanical applications due to enhanced responsivity. 


\section{Summary and outlook}

To conclude, we have surveyed the research done in recent years with regards to electromechanical and piezoelectric effects in non-nitride III-V NWs which, due to their distinct crystalline nature as compared to the bulk, present tremendous scope for both fundamental investigations as well as applications. Several trends arise which could further direct the study in this topic: firstly, regarding the mechanical properties of WZ III-Vs, it seems that the theoretical predictions hold for the elastic modulus in NWs with diameters greater than $10 \mathrm{~nm}$; secondly, the unique geometry and crystalline properties of III-V NWs (relaxed mechanical constraints, growth in $\{111\}$ orientation or WZ phase) imply that enhanced electromechanical properties may arise and thus should encourage further work in this area. Nevertheless, several aspects are crucial for further understanding of III-V NW piezoelectricity: (i) the role of stacking faults on the mechanical properties, and on piezoelectricity (as charge traps etc.); (ii) the role of non-linear piezoelectricity in III-V NWs (54), (iii) the complexities related to piezoelectric III-V semiconductors, particularly due to being more conductive compared to III-Ns, and giving rise to issues such as surface states and surface oxides. In all cases, development of robust and reliable nanocharacterization tools capable of measuring spatially resolved electromechanical properties of nanoscale materials is crucial to drive the field forward.

Considering the above, and the vast interest in electronic and optoelectronic applications of III-V NWs, further examination of the piezoelectric effect is an interesting research topic from both a fundamental and practical point of view, and could have potential useful applications opening up additional avenues for device improvement, particularly in the emerging areas of piezotronics and photo-piezotronics. The study of piezoelectricity in non-nitride III-V materials is particularly attractive considering the vast knowledge base related to these materials in terms of processing, and in particular in the possible combinations with optical properties in wavelengths spanning the visible spectrum.

\section{Acknowledgments}

S.K-N and Y.C are grateful for financial support from the European Research Council through an ERC Starting Grant (Grant No. ERC-2014-STG-639526, NANOGEN).

\section{References}

1. Y. Calahorra, X. Guan, N. N. Halder, M. Smith, S. Cohen, D. Ritter, J. Penuelas and S. KarNarayan: Exploring piezoelectric properties of III-V nanowires using piezo-response force microscopy. Semiconductor Science and Technology 32, 074006 (2017)

2. M. H. Zhao, Z. L. Wang and S. X. Mao: Piezoelectric characterization of individual zinc oxide nanobelt probed by piezoresponse force microscope. Nano Letters 4, 587-590 (2004)

3. Z. L. Wang and J. H. Song: Piezoelectric nanogenerators based on zinc oxide nanowire arrays. Science 312, 242-246 (2006) 
4. X. D. Wang, J. Zhou, J. H. Song, J. Liu, N. S. Xu and Z. L. Wang: Piezoelectric field effect transistor and nanoforce sensor based on a single ZnO nanowire. Nano Letters 6, 2768-2772 (2006)

5. F. Bernardini, V. Fiorentini and D. Vanderbilt: Spontaneous polarization and piezoelectric constants of III-V nitrides. Physical Review B 56, 10024-10027 (1997)

6. J. Goniakowski, F. Finocchi and C. Noguera: Polarity of oxide surfaces and nanostructures. Reports on Progress in Physics 71, 016501 (2008)

7. C. Y. Nam, P. Jaroenapibal, D. Tham, D. E. Luzzi, S. Evoy and J. E. Fischer: Diameter-dependent electromechanical properties of GaN nanowires. Nano Letters 6, 153-158 (2006)

8. M. Minary-Jolandan, R. A. Bernal, I. Kujanishvili, V. Parpoil and H. D. Espinosa: Individual GaN Nanowires Exhibit Strong Piezoelectricity in 3D. Nano Letters, 12, 970-976 (2012)

9. M. Z. Peng, Z. Li, C. H. Liu, Q. Zheng, X. Q. Shi, M. Song, Y. Zhang, S. Y. Du, J. Y. Zhai and Z. L. Wang: High-Resolution Dynamic Pressure Sensor Array Based on Piezo-phototronic Effect Tuned Photoluminescence Imaging. Acs Nano 9, 3143-3150 (2015)

10. N. Jamond, P. Chretien, F. Houze, L. Lu, L. Largeau, O. Maugain, L. Travers, J. C. Harmand, F. Glas, E. Lefeuvre, M. Tchernycheva and N. Gogneau: Piezo-generator integrating a vertical array of GaN nanowires. Nanotechnology 27, 325403 (2016)

11. Z. L. Wang: Piezopotential gated nanowire devices: Piezotronics and piezo-phototronics. Nano Today 5, 540-552 (2010)

12. Z. L. Wang: Piezotronic and Piezophototronic Effects. Journal of Physical Chemistry Letters, 1, 1388-1393 (2010)

13. J. Zhou, Y. D. Gu, P. Fei, W. J. Mai, Y. F. Gao, R. S. Yang, G. Bao and Z. L. Wang: Flexible piezotronic strain sensor. Nano Letters 8, 3035-3040 (2008)

14. X. Yang, L. Dong, C. Shan, J. Sun, N. Zhang, S. Wang, M. Jiang, B. Li, X. Xie and D. Shen: Piezophototronic-Effect-Enhanced Electrically Pumped Lasing. Advanced Materials 29, 1602832 (2017)

15. X. N. Wen, W. Z. Wu, C. F. Pan, Y. F. Hu, Q. Yang and Z. L. Wang: Development and progress in piezotronics. Nano Energy 14, 276-295 (2015)

16. N. P. Dasgupta, J. Sun, C. Liu, S. Brittman, S. C. Andrews, J. Lim, H. Gao, R. Yan and P. Yang: 25th Anniversary Article: Semiconductor Nanowires Synthesis, Characterization, and Applications. Advanced Materials 26, 2137-2184 (2014)

17. X. F. Duan, Y. Huang, Y. Cui, J. F. Wang and C. M. Lieber: Indium phosphide nanowires as building blocks for nanoscale electronic and optoelectronic devices. Nature 409, 66-69 (2001)

18. P. Krogstrup, H. I. Jorgensen, M. Heiss, O. Demichel, J. V. Holm, M. Aagesen, J. Nygard and A. F. I. Morral: Single-nanowire solar cells beyond the Shockley-Queisser limit. Nature Photonics 7, 306$310(2013)$

19. J. Singh: Electronic and optoelectronic properties of semiconductor structures. Cambridge University Press, New York (2003)

20. C. Wood, D. Jena: Polarization Effects in Semiconductors From Ab InitioTheory to Device Applications. In: Springer Science+Business Media, LLC, Boston, MA (2008) www.springer.com/gb/book/9780387368313

21. T. Hayakawa, M. Kondo, T. Suyama, K. Takahashi, S. Yamamoto and T. Hijikata: REDUCTION IN THRESHOLD CURRENT-DENSITY OF QUANTUM-WELL LASERS GROWN BY MOLECULAR-BEAM EPITAXY ON 0.5-DEGREES MISORIENTED (111)B SUBSTRATES. Japanese Journal of Applied Physics Part 2-Letters 26, L302-L305 (1987)

22. J. Nedbal and E. Klier: PIEZOELECTRIC RESONATORS OF SEMIINSULATING GAAS. Physica Status Solidi A-Applied Research 148, 329-340 (1995)

23. E. Klier and J. Nedbal: PIEZOELECTRIC RESONATORS OF INPFE. Czechoslovak Journal of Physics 44, 575-584 (1994)

24. T. Hanada: Basic Properties of $\mathrm{ZnO}, \mathrm{GaN}$, and Related Materials. Oxide and Nitride Semiconductors: Processing, Properties, and Applications 12, 1-19 (2009) 
25. P. Lawaetz: INTERNAL STRAIN IN ZINCBLENDE AND WURTZITE CRYSTALS. Physica Status Solidi B-Basic Research 57, 535-544 (1973)

26. M. C. Y. Huang, K. B. Cheng, Y. Zhou, B. Pesala, C. J. Chang-Hasnain and A. P. Pisano: Demonstration of piezoelectric actuated GaAs-based MEMS tunable VCSEL. leee Photonics Technology Letters 18, 1197-1199 (2006)

27. K. Hjort, J. Soderkvist and J. A. Schweitz: GALLIUM-ARSENIDE AS A MECHANICAL MATERIAL. Journal of Micromechanics and Microengineering 4, 1-13 (1994)

28. J. M. Hernandez, I. Izpura, E. Calleja and E. Munoz: PIEZOELECTRIC-INDUCED CURRENT ASYMMETRY IN 111 INGAAS/INALAS RESONANT-TUNNELING DIODES FOR MICROWAVE MIXING. Applied Physics Letters 63, 773-775 (1993)

29. I. H. Campbell, M. D. Joswick, D. L. Smith and R. H. Miles: OBSERVATION OF PIEZOELECTRIC EFFECTS IN STRAINED RESONANT-TUNNELING STRUCTURES GROWN ON (111)B GAAS. Applied Physics Letters 66, 988-990 (1995)

30. M. Kusaka: ELECTRICAL-PROPERTIES OF METAL PIEZO-ELECTRIC SEMICONDUCTOR INTERFACE UNDER STRESS. Surface Science 78, 209-219 (1978)

31. M. Kusaka, N. Hiraoka, M. Hirai and S. Okazaki: ELECTRICAL-PROPERTIES OF P-TYPE GAP SCHOTTKY-BARRIER UNDER STRESS. Surface Science 91, 264-270 (1980)

32. M. D. Gerngross, V. Sprincean, M. Leisner, J. Carstensen, H. Foll and I. Tiginyanu: Porous InP as Piezoelectric Component in Magnetoelectric Composite Sensors. Processes at the SemiconductorSolution Interface 4, 35, 67-72 (2011)

33. S. Cha, S. M. Kim, H. Kim, J. Ku, J. I. Sohn, Y. J. Park, B. G. Song, M. H. Jung, E. K. Lee, B. L. Choi, J. J. Park, Z. L. Wang, J. M. Kim and K. Kim: Porous PVDF As Effective Sonic Wave Driven Nanogenerators. Nano Letters 11, 5142-5147 (2011)

34. D. Cutaia, K. E. Moselund, H. Schmid, M. Borg, A. Olziersky, H. Riel and leee: Complementary III-V Heterojunction Lateral NW Tunnel FET Technology on Si. 2016 leee Symposium on VIsi Technology (2016)

35. J. J. Gu, O. Koybasi, Y. Q. Wu and P. D. Ye: III-V-on-nothing metal-oxide-semiconductor fieldeffect transistors enabled by top-down nanowire release process: Experiment and simulation. Applied Physics Letters 99, 112113 (2011)

36. S. A. Fortuna and X. L. Li: Metal-catalyzed semiconductor nanowires: a review on the control of growth directions. Semiconductor Science and Technology 25, 024005 (2010)

37. K. A. Dick, P. Caroff, J. Bolinsson, M. E. Messing, J. Johansson, K. Deppert, L. R. Wallenberg and L. Samuelson: Control of III-V nanowire crystal structure by growth parameter tuning. Semiconductor Science and Technology 25, 024009 (2010)

38. K. A. Dick and P. Caroff: Metal-seeded growth of III-V semiconductor nanowires: towards goldfree synthesis. Nanoscale 6, 3006-3021 (2014)

39. J. Noborisaka, J. Motohisa and T. Fukui: Catalyst-free growth of GaAs nanowires by selectivearea metalorganic vapor-phase epitaxy. Applied Physics Letters 86, 213102 (2005)

40. C. Colombo, D. Spirkoska, M. Frimmer, G. Abstreiter and A. F. I. Morral: Ga-assisted catalystfree growth mechanism of GaAs nanowires by molecular beam epitaxy. Physical Review B 77, 155326 (2008)

41. D. Dalacu, A. Kam, D. G. Austing, X. H. Wu, J. Lapointe, G. C. Aers and P. J. Poole: Selectivearea vapour-liquid-solid growth of InP nanowires. Nanotechnology 20, 395602 (2009)

42. A. Kelrich, Y. Calahorra, Y. Greenberg, A. Gavrilov, S. Cohen and D. Ritter: Shadowing and mask opening effects during selective-area vapor-liquid-solid growth of InP nanowires by metalorganic molecular beam epitaxy. Nanotechnology 24, 475302 (2013)

43. P. Mohan, J. Motohisa and T. Fukui: Realization of conductive InAs nanotubes based on latticemismatched InP/InAs core-shell nanowires. Applied Physics Letters 88, 013110 (2006)

44. S. Lehmann, J. Wallentin, D. Jacobsson, K. Deppert and K. A. Dick: A General Approach for Sharp Crystal Phase Switching in InAs, GaAs, InP, and GaP Nanowires. Using Only Group V Flow. Nano Letters 13, 4099-4105 (2013) 
45. A. Kelrich, O. Sorias, Y. Calahorra, Y. Kauffmann, R. Gladstone, S. Cohen, M. Orenstein and D. Ritter: InP Nanoflag Growth from a Nanowire Template by in Situ Catalyst Manipulation. Nano Letters 16, 2837-2844 (2016)

46. Y. Calahorra, A. Kelrich, S. Cohen and D. Ritter: Catalyst shape engineering for anisotropic cross-sectioned nanowire growth. Scientific Reports 7, 40891 (2017)

47. H. J. Joyce, J. Wong-Leung, Q. Gao, H. H. Tan and C. Jagadish: Phase Perfection in Zinc Blende and Wurtzite III-V Nanowires Using Basic Growth Parameters. Nano Letters 10, 908-915 (2010)

48. A. Kelrich, V. G. Dubrovskii, Y. Calahorra, S. Cohen and D. Ritter: Control of morphology and crystal purity of InP nanowires by variation of phosphine flux during selective area MOMBE. Nanotechnology 26, 085303 (2015)

49. D. Spirkoska, J. Arbiol, A. Gustafsson, S. Conesa-Boj, F. Glas, I. Zardo, M. Heigoldt, M. H. Gass, A. L. Bleloch, S. Estrade, M. Kaniber, J. Rossler, F. Peiro, J. R. Morante, G. Abstreiter, L. Samuelson and A. F. I. Morral: Structural and optical properties of high quality zinc-blende/wurtzite GaAs nanowire heterostructures. Physical Review B 80, 245325 (2009)

50. S. O. R. Moheimani, A. J. Fleming and SpringerLink (Online service): Piezoelectric Transducers for Vibration Control and Damping. In: Advances in industrial control,. Springer-Verlag London Limited,, London (2006) www.springer.com/gb/book/9781846283314

51. F. Boxberg, N. Sondergaard and H. Q. Xu: Elastic and Piezoelectric Properties of Zincblende and Wurtzite Crystalline Nanowire Heterostructures. Advanced Materials, 24(34), 4692-4706 (2012)

52. H. T. Mengistu and A. Garcia-Cristobal: The generalized plane piezoelectric problem: Theoretical formulation and application to heterostructure nanowires. International Journal of Solids and Structures 100, 257-269 (2016)

53. S. H. Park and S. L. Chuang: Comparison of zinc-blende and wurtzite GaN semiconductors with spontaneous polarization and piezoelectric field effects. Journal of Applied Physics 87, 353-364 (2000) 54. H. Y. S. Al-Zahrani, J. Pal, M. A. Migliorato, G. Tse and D. P. Yu: Piezoelectric field enhancement in III-V core-shell nanowires. Nano Energy, 14, 382-391 (2015)

55. D. Berlincourt, L. R. Shiozawa and H. Jaffe: ELECTROELASTIC PROPERTIES OF SULFIDES, SELENIDES, AND TELLURIDES OF ZINC AND CADMIUM. Physical Review 129, 1009 (1963)

56. S. Q. Wang and H. Q. Ye: First-principles study on elastic properties and phase stability of III-V compounds. Physica Status Solidi B-Basic Research 240, 45-54 (2003)

57. R. X. Yan, D. Gargas and P. D. Yang: Nanowire photonics. Nature Photonics 3, 569-576 (2009)

58. Y. Calahorra, O. Shtempluck, V. Kotchetkov and Y. E. Yaish: Young's Modulus, Residual Stress, and Crystal Orientation of Doubly Clamped Silicon Nanowire Beams. Nano Letters 15, 2945-2950 (2015)

59. A. Heidelberg, L. T. Ngo, B. Wu, M. A. Phillips, S. Sharma, T. I. Kamins, J. E. Sader and J. J. Boland: A generalized description of the elastic properties of nanowires. Nano Letters 6, 1101-1106 (2006)

60. M. Dunaevskiy, P. Geydt, E. Lahderanta, P. Alekseev, T. Haggren, J. P. Kakko, H. Jiang and H. Lipsanen: Young's Modulus of Wurtzite and Zinc Blende InP Nanowires. Nano Letters 17, 3441-3446 (2017)

61. P. A. Alekseev, M. S. Dunaevskii, A. V. Stovpyaga, M. Lepsa and A. N. Titkov: Measurement of Young's modulus of GaAs nanowires growing obliquely on a substrate. Semiconductors 46, 641-646 (2012)

62. Y. B. Wang, L. F. Wang, H. J. Joyce, Q. A. Gao, X. Z. Liao, Y. W. Mai, H. H. Tan, J. Zou, S. P. Ringer, H. J. Gao and C. Jagadish: Super Deformability and Young's Modulus of GaAs Nanowires. Advanced Materials 23, 1356-1360 (2011)

63. B. Chen, Q. Gao, Y. Wang, X. Liao, Y. W. Mai, H. H. Tan, J. Zou, S. P. Ringer and C. Jagadish: Anelastic Behavior in GaAs Semiconductor Nanowires. Nano Letters 13, 3169-3172 (2013)

64. Y. J. Chen, T. Burgess, X. H. An, Y. W. Mai, H. H. Tan, J. Zou, S. P. Ringer, C. Jagadish and X. Z. Liao: Effect of a High Density of Stacking Faults on the Young's Modulus of GaAs Nanowires. Nano Letters 16, 1911-1916 (2016) 
65. P. A. Mante, S. Lehmann, N. Anttu, K. A. Dick and A. Yartsev: Nondestructive Complete Mechanical Characterization of Zinc Blende and Wurtzite GaAs Nanowires Using Time-Resolved PumpProbe Spectroscopy. Nano Letters 16, 4792-4798 (2016)

66. R. M. Martin: RELATION BETWEEN ELASTIC TENSORS OF WURTZITE AND ZINCBLENDE STRUCTURE MATERIALS. Physical Review B 6, 4546-4553 (1972)

67. X. Li, X. L. Wei, T. T. Xu, Z. Y. Ning, J. P. Shu, X. Y. Wang, D. Pan, J. H. Zhao, T. Yang and Q. Chen: Mechanical properties of individual InAs nanowires studied by tensile tests. Applied Physics Letters 104, 103110 (2014)

68. R. Erdelyi, M. H. Madsen, G. Safran, Z. Hajnal, I. E. Lukacs, G. Fulop, S. Csonka, J. Nygard and J. Volk: In-situ mechanical characterization of wurtzite InAs nanowires. Solid State Communications 152, 1829-1833 (2012)

69. Y. M. Niquet and D. C. Mojica: Quantum dots and tunnel barriers in InAsOInP nanowire heterostructures: Electronic and optical properties. Physical Review B 77, 115316 (2008)

70. P. E. Faria and G. M. Sipahi: Band structure calculations of InP wurtzite/zinc-blende quantum wells. Journal of Applied Physics 112, 103716 (2012)

71. M. Holm, M. E. Pistol and C. Pryor: Calculations of the electronic structure of strained InAs quantum dots in InP. Journal of Applied Physics 92, 932-936 (2002)

72. G. Bester and A. Zunger: Cylindrically shaped zinc-blende semiconductor quantum dots do not have cylindrical symmetry: Atomistic symmetry, atomic relaxation, and piezoelectric effects. Physical Review B 71, 045318 (2005)

73. M. Hocevar, L. T. T. Giang, R. Songmuang, M. den Hertog, L. Besombes, J. Bleuse, Y. M. Niquet and N. T. Pelekanos: Residual strain and piezoelectric effects in passivated GaAs/AlGaAs core-shell nanowires. Applied Physics Letters 102, 191103 (2013)

74. M. Zervos and L. F. Feiner: Electronic structure of piezoelectric double-barrier InAs/InP/InAs/InP/InAs(111) nanowires. Journal of Applied Physics 95, 281-291 (2004)

75. R. Anufriev, N. Chauvin, H. Khmissi, K. Naji, G. Patriarche, M. Gendry and C. Bru-Chevallier: Piezoelectric effect in InAs/InP quantum rod nanowires grown on silicon substrate. Applied Physics Letters 104, 183101 (2014)

76. K. Moratis, S. L. Tan, S. Germanis, C. Katsidis, M. Androulidaki, K. Tsagaraki, Z. Hatzopoulos, F. Donatini, J. Cibert, Y. M. Niquet, H. Mariette and N. Pelekanos: Strained GaAs/InGaAs Core-Shell Nanowires for Photovoltaic Applications. Nanoscale Research Letters 11, 1-7 (2016)

77. I. J. Chen, S. Lehmann, M. Nilsson, P. Kivisaari, H. Linke, K. A. Dick and C. Thelandert: Conduction Band Offset and Polarization Effects in InAs Nanowire Polytype Junctions. Nano Letters 17, 902-908 (2017)

78. N. Chauvin, A. Mavel, G. Patriarche, B. Masenelli, M. Gendry and D. Machon: PressureDependent Photoluminescence Study of Wurtzite InP Nanowires. Nano Letters 16, 2926-2930 (2016)

79. X. Li, X. L. Wei, T. T. Xu, D. Pan, J. H. Zhao and Q. Chen: Remarkable and Crystal-StructureDependent Piezoelectric and Piezoresistive Effects of InAs Nanowires. Advanced Materials 27, 2852 (2015)

80. K. Zheng, Z. Zhang, Y. B. Hu, P. P. Chen, W. Lu, J. Drennan, X. D. Han and J. Zou: Orientation Dependence of Electromechanical Characteristics of Defect-free InAs Nanowires. Nano Letters 16, 1787-1793 (2016)

81. J. H. Lee, M. W. Pin, S. J. Choi, M. H. Jo, J. C. Shin, S. G. Hong, S. M. Lee, B. Cho, S. J. Ahn, N. W. Song, S. H. Yi and Y. H. Kim: Electromechanical Properties and Spontaneous Response of the Current in InAsP Nanowires. Nano Letters 16, 6738-6745 (2016)

82. G. Signorello, S. Sant, N. Bologna, M. Schraff, U. Drechsler, H. Schmid, S. Wirths, M. D. Rossell, A. Schenk and H. Riel: Manipulating Surface States of III-V Nanowires with Uniaxial Stress. Nano Letters 17, 2816-2824 (2017)

83. G. Signorello, E. Lortscher, P. A. Khomyakov, S. Karg, D. L. Dheeraj, B. Gotsmann, H. Weman and $\mathrm{H}$. Riel: Inducing a direct-to-pseudodirect bandgap transition in wurtzite GaAs nanowires with uniaxial stress. Nature Communications 5, 3655 (2014) 
84. G. Signorello, S. Karg, M. T. Bjork, B. Gotsmann and H. Riel: Tuning the Light Emission from GaAs Nanowires over 290 meV with Uniaxial Strain. Nano Letters 13, 917-924 (2013)

85. I. P. Soshnikov, D. E. Afanas'ev, V. A. Petrov, G. E. Cirlin, A. D. Bouravlev, Y. B. Samsonenko, A. Khrebtov, E. M. Tanklevskaya and I. A. Seleznev: Piezoelectric effect in GaAs nanowires. Semiconductors 45, 1082-1084 (2011)

86. V. Lysak, I. P. Soshnikov, E. Lahderanta and G. E. Cirlin: Piezoelectric effect in GaAs nanowires: experiment and theory. Physica Status Solidi-Rapid Research Letters 10, 172-175 (2016)

87. V. V. Lysak, I. P. Soshnikov, E. Lahderanta and G. E. Cirlin: Piezoelectric effect in wurtzite GaAs nanowires: Growth, characterization, and electromechanical 3D modeling. Physica Status Solidi aApplications and Materials Science 213, 3014-3019 (2016)

88. Y. Calahorra, M. Smith, A. Datta, H. Benisty and S. Kar-Narayan: Mapping piezoelectric response in nanomaterials using a dedicated non destructive scanning probe technique. Nanoscale 9 , 8, 19290-19297 (2017)

89. A. Gruverman and S. V. Kalinin: Piezoresponse force microscopy and recent advances in nanoscale studies of ferroelectrics. Journal of Materials Science 41, 107-116 (2006)

90. N. Tiwary, R. Sarkar, V. R. Rao, A. Laha and leee: Piezoresponse Force Microscopy (PFM) characterization of GaN nanowires grown by Plasma Assisted Molecular Beam Epitaxy (PA-MBE). 2016 Joint leee International Symposium on the Applications of Ferroelectrics, European Conference on Application of Polar Dielectrics, and Piezoelectric Force Microscopy Workshop (Isaf/Ecapd/Pfm) (2016) 91. R. Agrawal and H. D. Espinosa: Giant Piezoelectric Size Effects in Zinc Oxide and Gallium Nitride Nanowires. A First Principles Investigation. Nano Letters 11, 786-790 (2011)

92. M. T. Hoang, J. Yvonnet, A. Mitrushchenkov and G. Chambaud: First-principles based multiscale model of piezoelectric nanowires with surface effects. Journal of Applied Physics 113, 014309 (2013)

93. I. L. Guy, S. Muensit and E. M. Goldys: Extensional piezoelectric coefficients of gallium nitride and aluminum nitride. Applied Physics Letters 75, 4133-4135 (1999)

94. P. H. Wang, H. J. Du, S. N. Shen, M. S. Zhang and B. Liu: Preparation and characterization of ZnO microcantilever for nanoactuation. Nanoscale Research Letters 7, 1-5 (2012) 
(a)

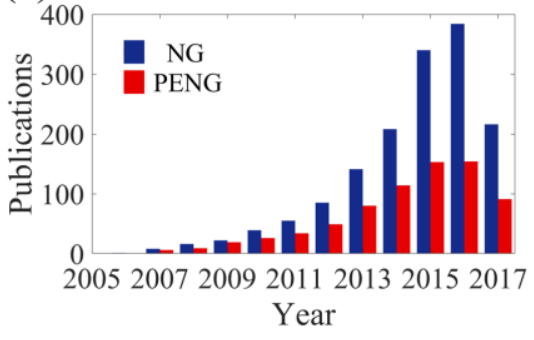

(b)

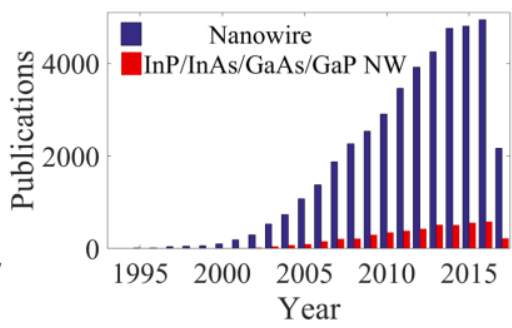

(c)

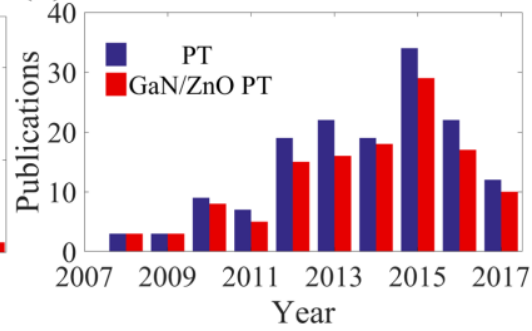

Figure 1. Web of Science topical publication data: a) "Nanogenerator" vs.

"Piezoelectric+Nanogenerator"; b) "Nanowire" vs. "InP+Nanowire OR InAs+Nanowire OR GaAs+Nanowire OR GaP+Nanowire"; c) "Piezotronic" vs. "Piezotronic+GaN OR

Piezotronic+ZnO".

(a)

Metal compressive strain

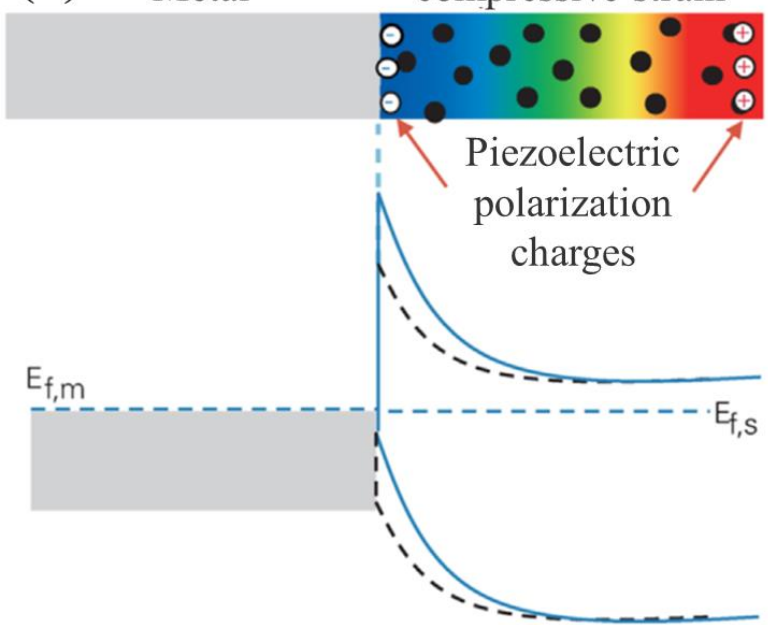

(b)

Metal Tensile strain

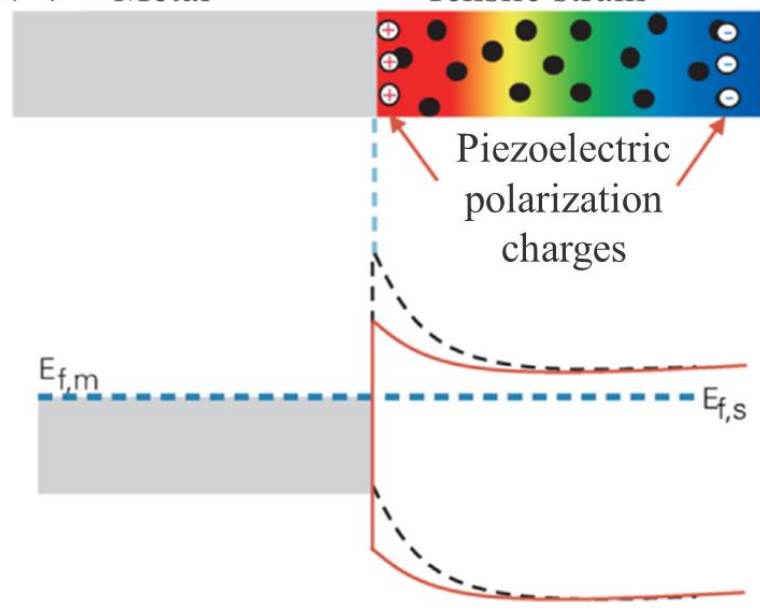

Figure 2. Schematic describing the piezotronic effect: a) and b) show a piezoelectric semiconductor in contact with a metal, such that compression (a) and tension (b) of the semiconductor give rise to uncompensated surface charge at the Schottky interface - modulating the barrier height. Reprinted with permission from Elsevier(15). 


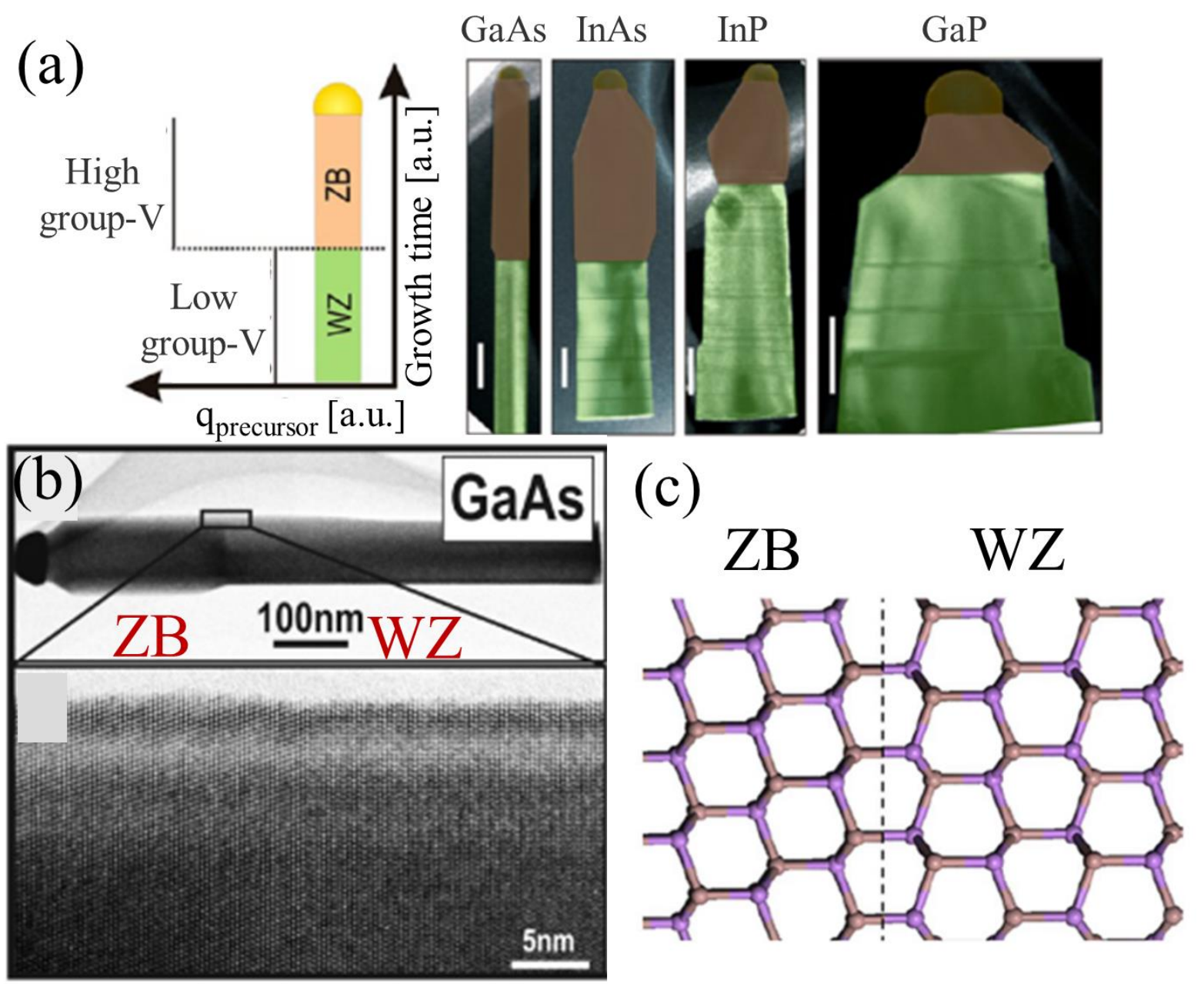

Figure 3. Crystal phase control in III-V NWs: a) switching from WZ to ZB by increasing growth precursor flow for GaAs, InAs, InP, GaP. Reprinted with permission from ACS (44) b) HRTEM showing the phase purity around the GaAs crystal phase interface. Reprinted with permission from ACS (44); c) schematic corresponding to the interface in (c). 

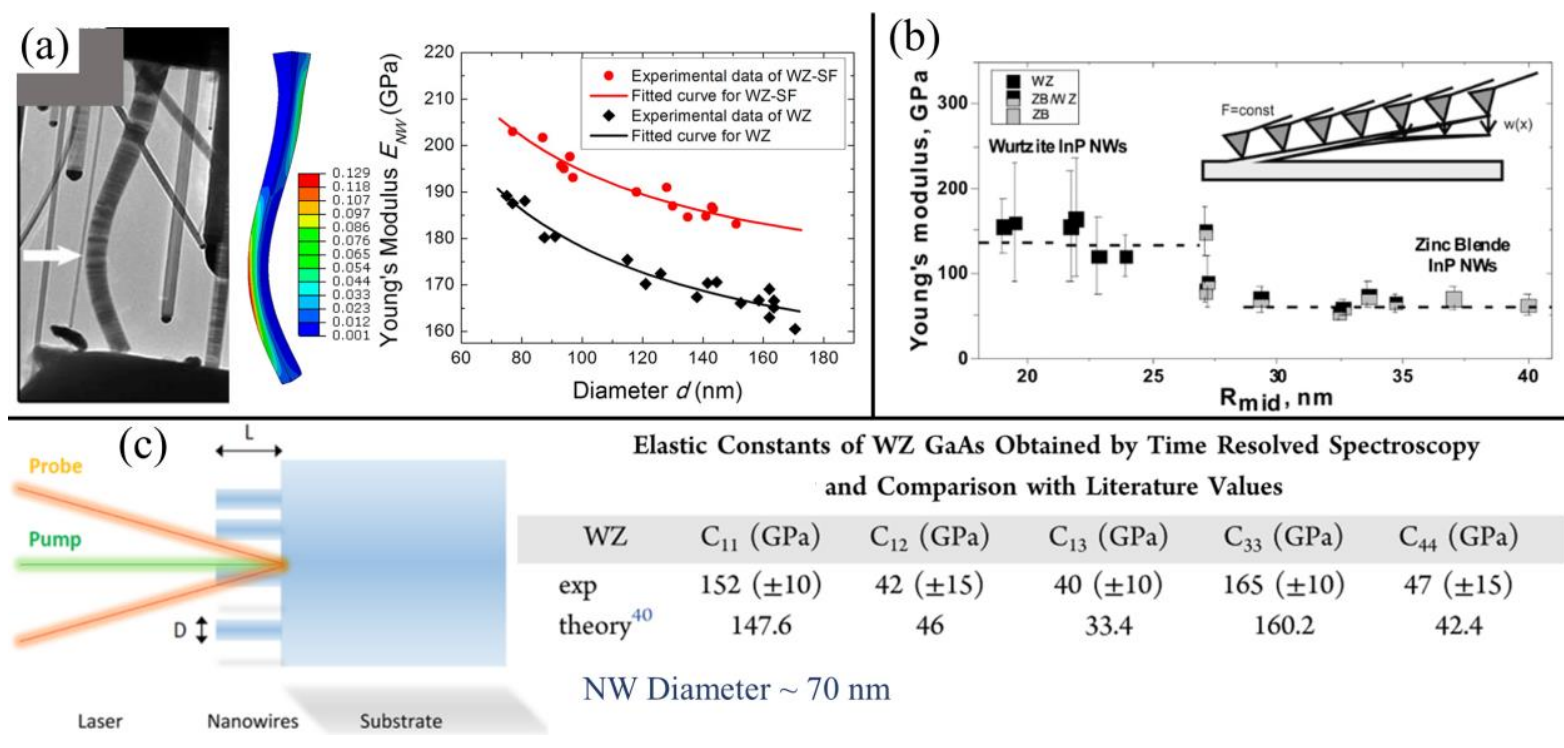

Figure 4. Experimentally obtained elastic moduli values for WZ GaAs and InP NWs. a) TEM and finite-element analogue for compression of GaAs NWs (left) and axial Young's modulus results for NWs w/wo stacking faults (right). Reprinted with permission from ACS (64); b) schematic of inclined InP NW bending (inset) and axial Young's modulus results for NW w/wo stacking faults. Reprinted with permission from ACS (60); c) schematic of the optical pump-probe setup (left) and extracted vs. theoretical stiffness coefficients for WZ GaAs NWs. Reprinted with permission from ACS (65). 

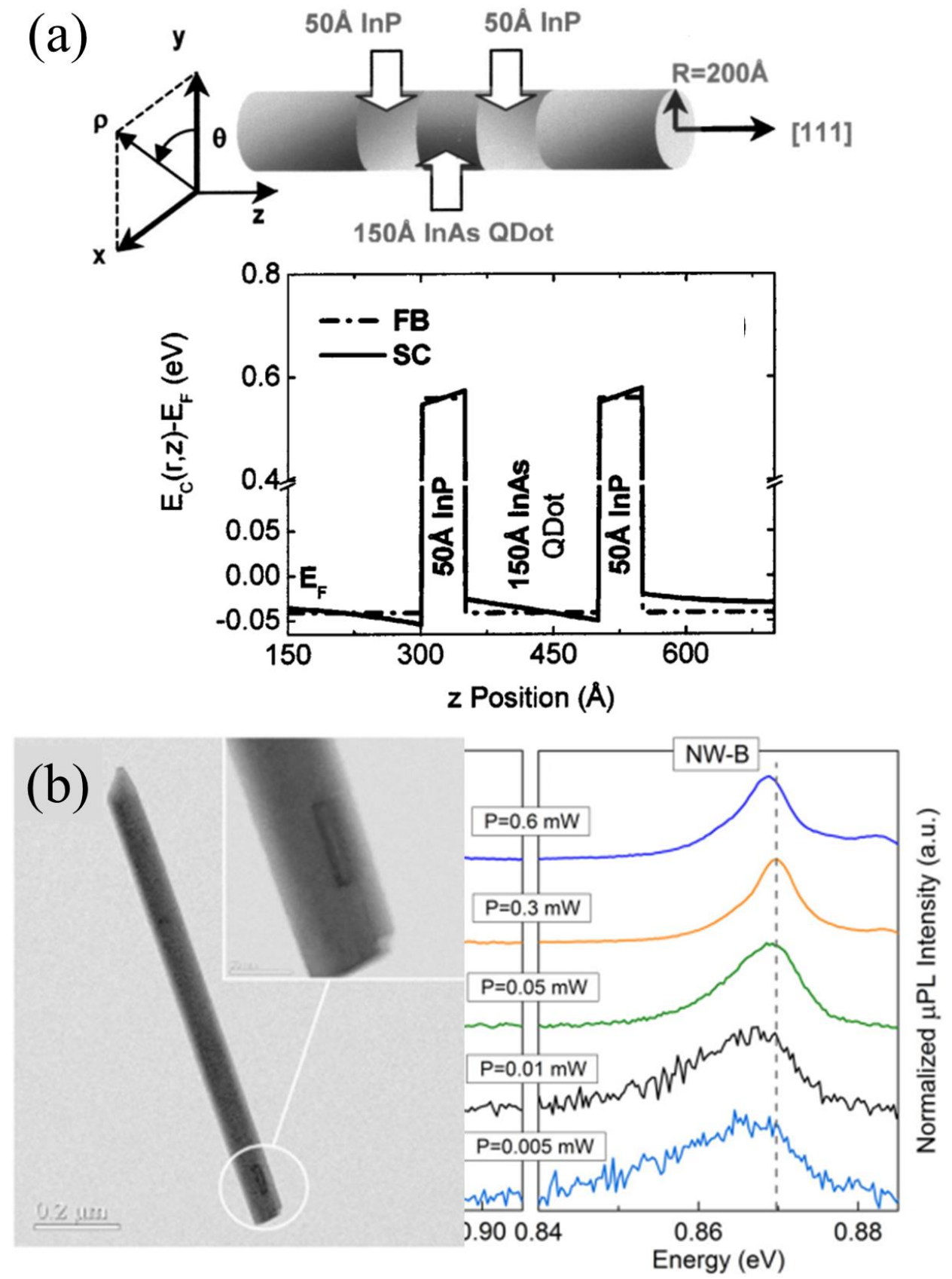

Figure 5. Internal piezoelectric effects: a) schematic structure (top) and calculated contuction band of the InP/InAs double barrier resonant tunneling diode structure, with self-consistent solution containing the piezoelectirc fields in the structure. Reprinted with premission from AIP(74); b) TEM showing an InAs quantum rod embedded within an InP NW (left) and Stark effect characteristic low excitation blue shift measured in these structures (right). Reprinted with premission from AIP (75). 


\section{(a)}
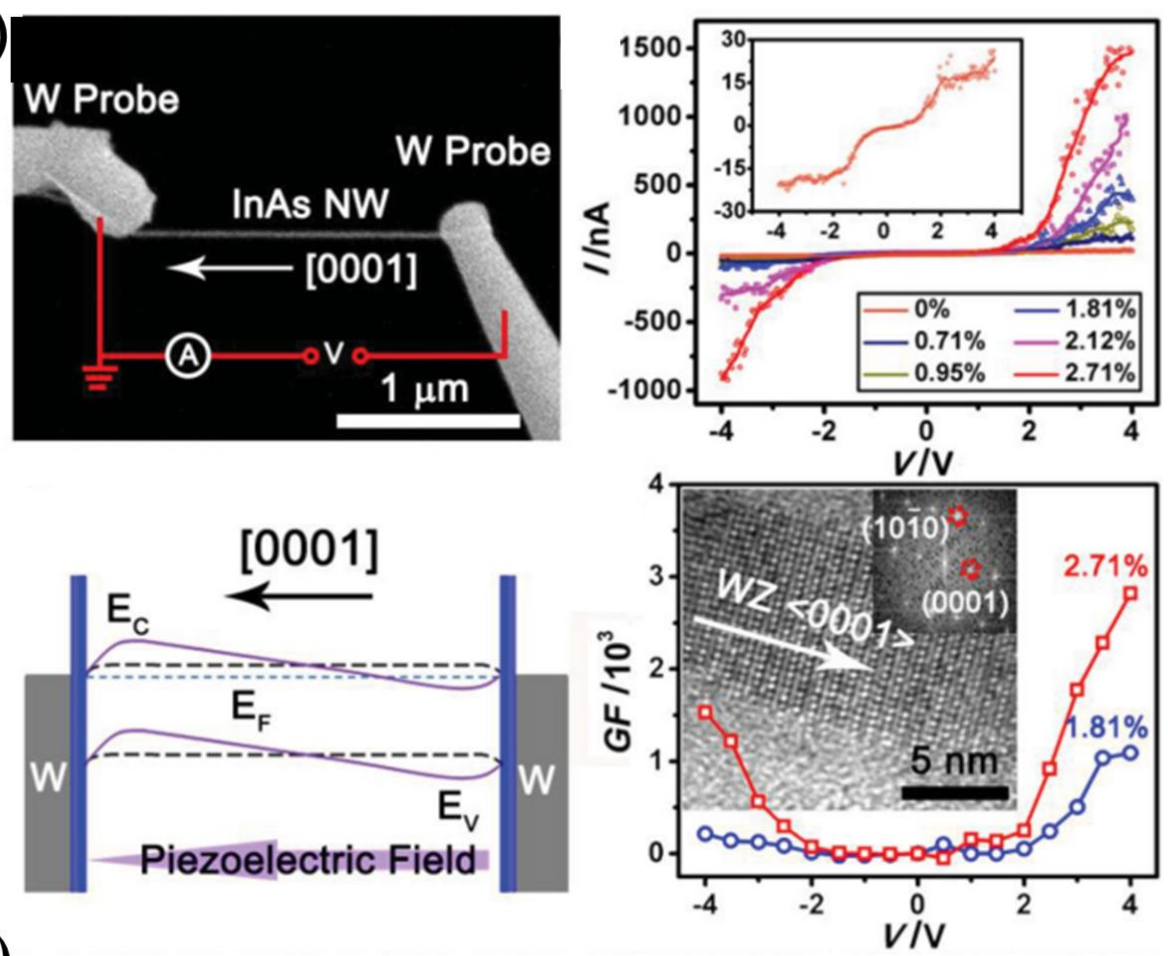

(b)
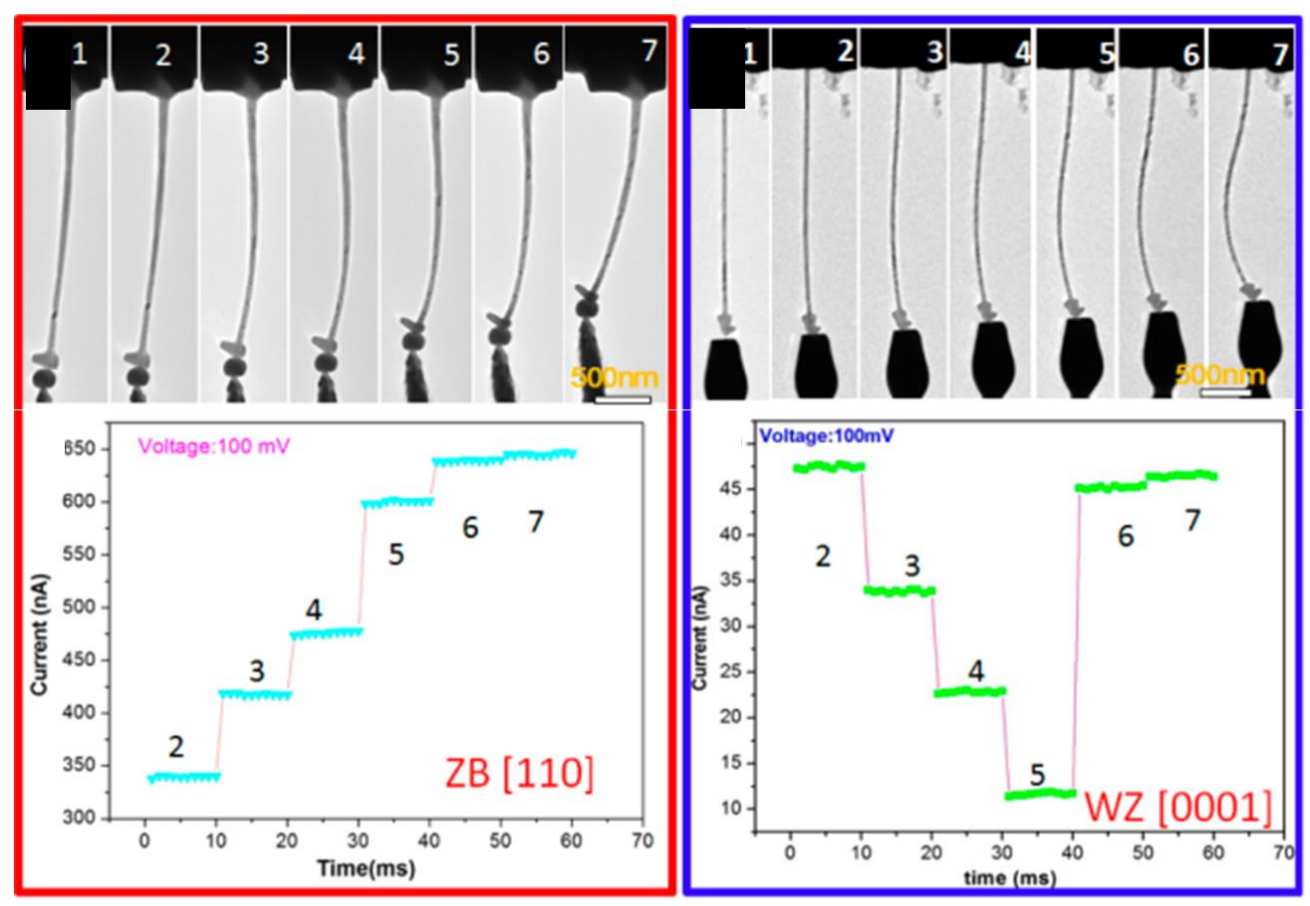

Figure 6. Externally applied electromechanical effects: a) WZ InAs NW piezotronics. Electron microscopy based stretching scheme (top left), I-V curves for various strain levels (top right), schematic band diagram (bottom left) and electromechanical gauge factor for two strain levels (bottom right). Reprinted with permission from Wiley (79); b) ZB/WZ InAs NW piezoresistive effect. Left and right show compression experiments on ZB and WZ NWs correspondingly. The graphs below show the consequent strain related current levels. Reprinted with permission from ACS (80). 


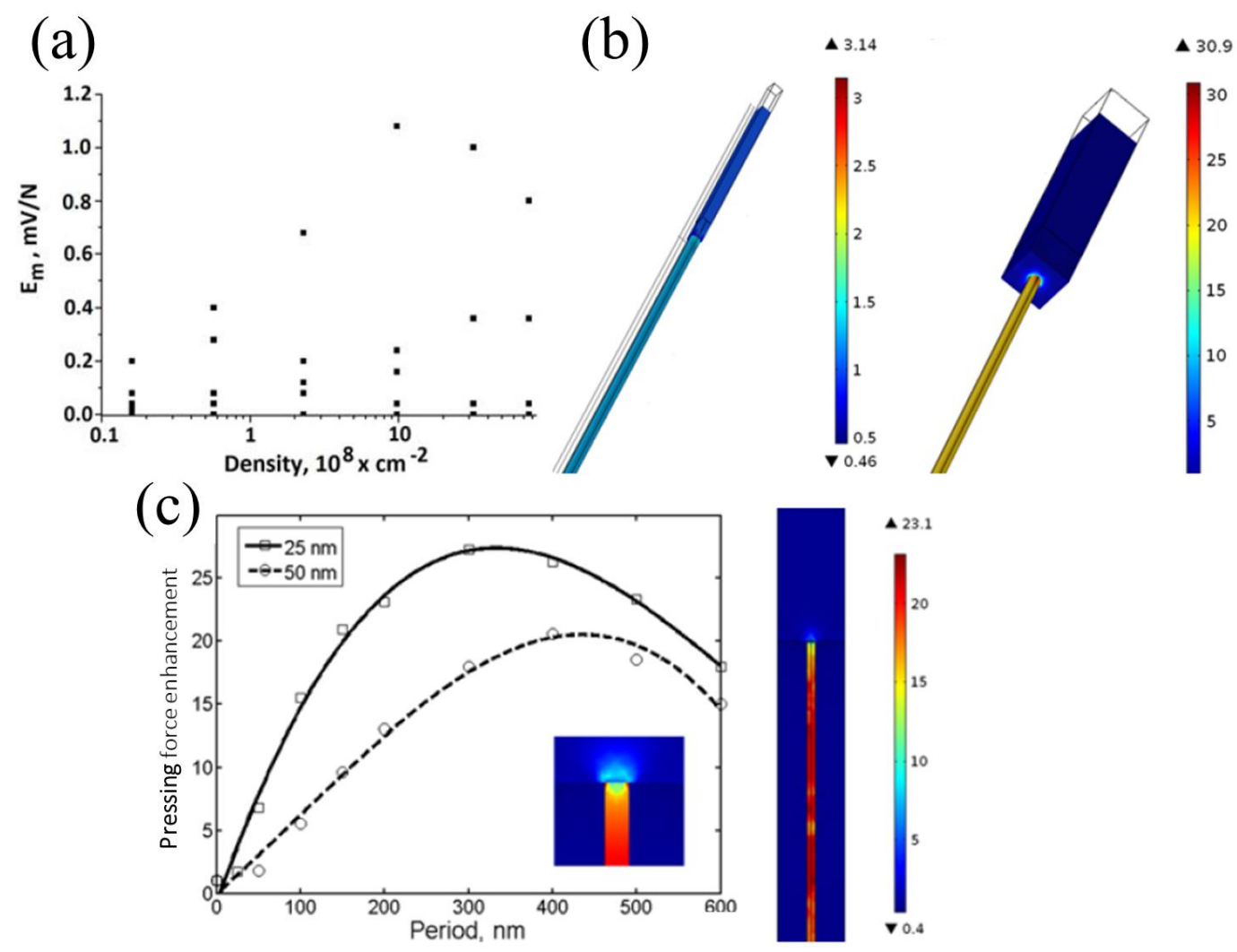

Figure 7. The piezoelectric properties of GaAs NW ensembles: a) experimentally measured electromechanical coupling as a function of NW density; b) finite element simulation of the pressure force enhancement effect; c) simulation of the intra-NW medium, recreating the NW density effect. Reprinted with permission from Wiley (86).

(a)

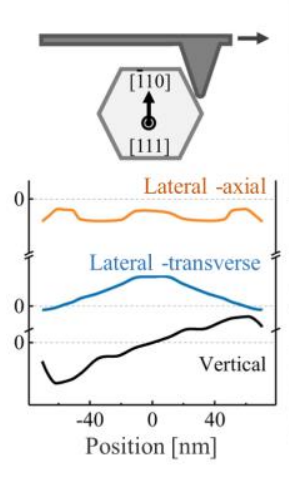

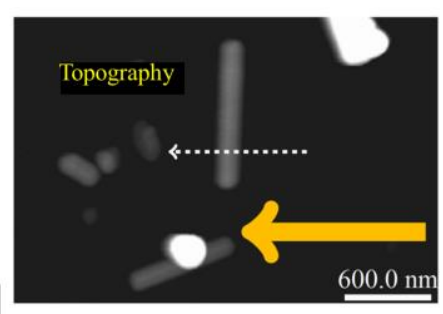

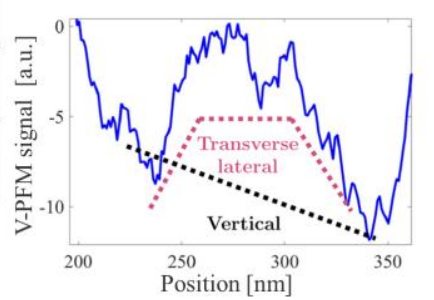

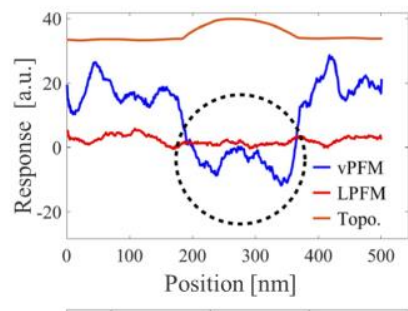

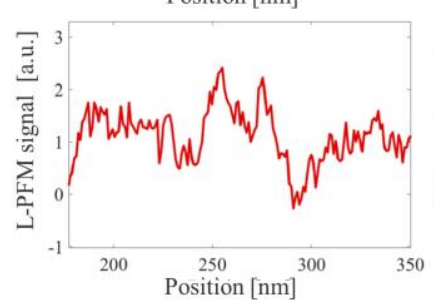

(b)
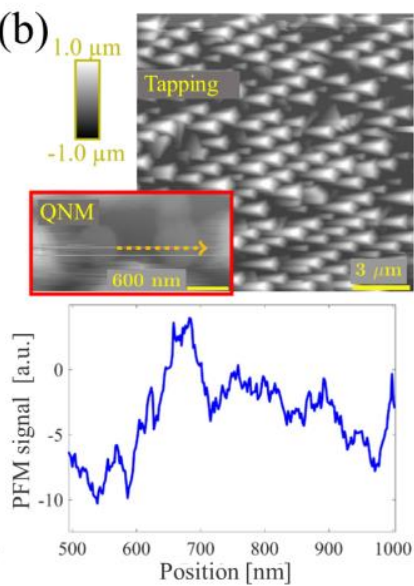

Figure 8. The piezoelectric properties single III-V NWs probed by PFM: a) Horizontally measured ZB GaAs NW (scheme - top left, and AFM capture - top middle), where the results from finite element simulations (left) successfully recreated the experimentally obtained lateral and vertical PFM signals (bottom middle and right); b) vertically aligned WZ InP NWs (AFM capture - top, PFM scanned NWs - inset) and the resulting PFM from the substrate and the NW (bottom), used to extract a $d_{33}$ value. Reprinted with permission from IOP (1). 
Table I. The piezoelectric coefficients of III-V materials for ZB $<111>$ orientation, and for WZ.

\begin{tabular}{|c|c|c|c|c|c|c|c|c|c|}
\hline & \multicolumn{3}{|c|}{$\overline{\mathrm{ZB}}$} & \multicolumn{6}{|c|}{$\overline{\mathrm{WZ}}$} \\
\hline & $\widetilde{e_{14}}$ & $\widetilde{d_{14}}$ & $d_{33,<111>}$ & $\mathrm{e}_{33, \mathrm{QC}}$ & $\mathrm{d}_{33, \mathrm{QC}}$ & $\mathrm{d}_{31, \mathrm{QC}}$ & $\mathrm{e}_{33, \mathrm{AI}}$ & $d_{33, \mathrm{AI}}$ & $d_{31, \mathrm{AI}}$ \\
\hline \multirow{4}{*}{$\begin{array}{c}\mathrm{InP} \\
\mathrm{GaP} \\
\mathrm{GaAs} \\
\mathrm{InAs}\end{array}$} & 0.04 & 0.87 & 0.5 & 0.0231 & 0.22 & -0.11 & 0.59 & 5.42 & -2.4 \\
\hline & 0.10 & 1.43 & 0.82 & 0.0577 & 0.37 & -0.19 & 0.48 & 3.18 & -1.66 \\
\hline & 0.16 & 2.6 & 1.54 & 0.0924 & 0.73 & -0.36 & 0.32 & 2.55 & -1.317 \\
\hline & 0.05 & 1.25 & 0.72 & 0.0289 & 0.33 & -0.16 & 0.51 & 5.76 & -2.91 \\
\hline & \multicolumn{4}{|c|}{$d_{33}$} & \multicolumn{5}{|c|}{$d_{31}$} \\
\hline $\mathrm{GaN}$ & \multicolumn{4}{|c|}{$2.8-3.7(93)$} & \multicolumn{5}{|c|}{$-(1.4-1.9)(93)$} \\
\hline $\mathrm{ZnO}$ & \multicolumn{4}{|c|}{$10-12(2)$} & \multicolumn{5}{|c|}{$-(3.27-5.2)(94)$} \\
\hline
\end{tabular}

